OPEN ACCESS

Edited by:

Emily Keshner,

Temple University, United States

Reviewed by:

Pedro Ribeiro,

Universidade Federal do Rio de

Janeiro, Brazil

Matteo Bologna,

Sapienza Università di Roma, Italy

*Correspondence: Brett W. Fling

brett.fling@colostate.edu

Specialty section: This article was submitted to Movement Disorders, a section of the journal

Frontiers in Neurology

Received: 31 January 2018 Accepted: 20 March 2018

Published: 04 April 2018

Citation:

Fling BW, Curtze $C$ and Horak FB (2018) Gait Asymmetry in People With Parkinson's Disease Is Linked to Reduced Integrity of Callosal Sensorimotor Regions. Front. Neurol. 9:215.

doi: 10.3389/fneur.2018.00215

\section{Gait Asymmetry in People With Parkinson's Disease Is Linked to Reduced Integrity of Callosal Sensorimotor Regions}

\author{
Brett W. Fling ${ }^{1,2 *}$, Carolin Curtze ${ }^{3}$ and Fay B. Horak ${ }^{3,4}$ \\ ${ }^{1}$ Department of Health and Exercise Science, Colorado State University, Fort Collins, CO, United States, ${ }^{2}$ Molecular, Cellular \\ and Integrative Neuroscience Program, Colorado State University, Fort Collins, CO, United States, ${ }^{3}$ Department of \\ Neurology, Oregon Health \& Science University, Portland, OR, United States, ${ }^{4}$ Veterans Affairs Portland Health Care System, \\ Portland, OR, United States
}

Background: Individuals with Parkinson's disease (PD) often manifest significant temporal and spatial asymmetries of the lower extremities during gait, which significantly contribute to mobility impairments. While the neural mechanisms underlying mobility asymmetries within this population remain poorly understood, recent evidence points to altered microstructural integrity of white matter fiber tracts within the corpus callosum as potentially playing a substantial role.

Objectives: The purpose of this study was to quantify spatial and temporal gait asymmetries as well as transcallosal microstructural integrity of white matter fiber tracts connecting the primary and secondary sensorimotor cortices in people with PD and age-matched control participants.

Methods: Spatial and temporal gait asymmetry in the levodopa off state was assessed using an instrumented walkway. On the next day, diffusion-weighted images were collected to assess white matter microstructural integrity in transcallosal fibers connecting the homologous sensorimotor cortical regions.

Results: People with PD exhibited significantly more temporal and spatial gait asymmetry than healthy control subjects. Furthermore, people with PD had significantly reduced white matter microstructural integrity of transcallosal fibers connecting homologous regions of the pre-supplementary motor and supplementary motor areas (SMAs), but not the primary motor or somatosensory cortices. Finally, reduced transcallosal fiber tract integrity of the pre-SMA and S1 was associated with greater step length asymmetry in people with PD.

Conclusion: People with PD showed increased step length asymmetries and decreased microstructural integrity of callosal white matter tracts connecting the higher-order sensorimotor cortices (pre-SMA and SMA). The strong association between gait asymmetries and corpus collosum integrity, supports the hypothesis that reduced transcallosal structural connectivity is a significant mechanism underlying gait asymmetries in people with PD.

Keywords: MRI, diffusion-weighted imaging, gait, balance, transcallosal, mobility 


\section{INTRODUCTION}

Impaired walking ability is common in persons with Parkinson's disease (PD), typically manifesting as reduced gait velocity and step length, increased gait variability, and reduced automaticity (1). PD has also been associated with temporal and spatial asymmetries of the lower extremities during gait $(2,3)$. These lower extremity asymmetries significantly contribute to mobility impairments in neurologic populations who experience gait and balance dysfunction. Reduced coordination during gait (i.e., increased gait asymmetry) is associated with increased metabolic cost, postural instability, falls, and reduced quality of life in those living with PD or following a stroke (4-6). While significant asymmetries in lower extremity control typically arise from unilateral neurologic insult such as a stroke, spinal cord injury, or traumatic brain injury, the neural mechanisms underlying mobility asymmetries within people with PD remain poorly understood. Although sparsely investigated, recent work suggests that altered microstructural integrity of white matter fiber tracts within the corpus callosum may play(s) a role (7-9).

Transcallosal communication via the corpus callosum plays a key role in the production of integrated motor behavior to generate appropriate, coordinated motor responses on both sides of the body $(10,11)$. The primary motor cortices are connected to the contralateral muscles controlling movement and are also densely interconnected through the corpus callosum allowing for interhemispheric transfer of information. When precisely, bilaterally coordinating movements in time and space (e.g., walking or typing), activation of one limb has a cumulative, inhibitory effect on the ipsilateral motor cortex, obtained via interhemispheric communication $(12,13)$. The relationship between reduced transcallosal structural connectivity and impaired bimanual upper extremity control is clear and well studied $(10,11)$, but it remains unclear if these associations extend to bilateral control of the lower extremities as well. That is to say, reduced structural connectivity of the corpus callosum is common in $\operatorname{PD}(8,14,15)$, yet it remains to be tested how reduced transcallosal structure contributes to the lower limb asymmetries observed during gait and balance tasks.

The purpose of this study was to quantify spatial and temporal gait asymmetries (assessed via an instrumented walkway) as well as transcallosal microstructural integrity of white matter fiber tracts connecting the primary sensorimotor cortices and supplementary motor areas (SMAs) (assessed via diffusion-weighted imaging) in people with PD and age-matched control participants with no known neurologic conditions. Our overarching hypothesis was that those with PD would have increased spatial and temporal gait asymmetries during over-ground walking, associated with reduced sensorimotor corpus callosum structural connectivity compared with their age-matched counterparts.

\section{MATERIALS AND METHODS}

\section{Participant Demographics}

We recruited 39 people with idiopathic PD and 20 age-matched healthy controls (Table 1). The protocol was approved by the Institutional Review Board of Oregon Health and Science University. All subjects gave written informed consent in
TABLE 1 | Participant demographics.

\begin{tabular}{lcc}
\hline & Parkinson's disease & HC \\
\hline$n$ & 39 & 20 \\
Age (years) & $68.7(8.0)$ & $71.4(8.1)$ \\
Sex (M/F) & $26 / 13$ & $7 / 13$ \\
Disease duration (years) & $7.1(5.7)$ & \\
MDS-UPDRS III & $40.1(13.6)$ & \\
PIGD & $5.5(3.5)$ & $27.1(1.9)$ \\
Hoehn and Yahr & $2.4(0.6)$ & \\
MoCA & $24.4(4.1)$ & \\
Levodopa equivalent dose (mg) & $1,024(75-8,680)$ & \\
\hline
\end{tabular}

accordance with the Declaration of Helsinki. Clinical, mobility, and neuroimaging testing was performed over the course of two test sessions, separated by less than 1 week. All participants with PD were tested in the OFF medication state, that is, after withholding their dopaminergic medication for at least $12 \mathrm{~h}$.

\section{Mobility Assessment}

Participants walked at preferred gait speed three times over an $8-\mathrm{m}$ long instrumented walkway with an active area of $6 \mathrm{~m} \times 0.6 \mathrm{~m}$ sampling at a frequency of $60 \mathrm{~Hz}$ (GAITRite, CIR System, Havertown, PA, USA). Spatial and temporal asymmetry in percent was calculated as follows:

$$
\mid 1-\text { left/right } \mid{ }^{\star} 100 \text {, }
$$

for step length and step time, respectively.

\section{Image Acquisition}

Neuroimaging data were collected at the Oregon Health and Science University's Advanced Imaging Research Center on a 3.0 T Siemens Magnetom Tim Trio scanner with a 12-channel head coil. Collection parameters were similar to previous research conducted by our lab (16). Briefly, a structural, high-resolution T1-weighted MP-RAGE sequence was collected (orientation $=$ sagittal, echo time $=3.58 \mathrm{~ms}$, repetition time $=2,300 \mathrm{~ms}$, $256 \times 256$ matrix, resolution $1.0 \mathrm{~mm} \times 1.0 \mathrm{~mm} \times 1.1 \mathrm{~mm})$. In addition, high angular resolution diffusion images were also acquired using a 72-gradient direction, whole-brain echoplanar imaging sequence $(\mathrm{TR}=7,100 \mathrm{~ms}, \mathrm{TE}=112 \mathrm{~ms}$, field of view $=230 \mathrm{~mm} \times 230 \mathrm{~mm}, b$ value $=3,000 \mathrm{~s} / \mathrm{mm}^{2}$, isotropic voxel dimensions $=2.5 \mathrm{~mm}^{3}$ ) and 10 non-diffusion-weighted images where the $b$ value was equal to 0 .

\section{Diffusion Tensor Imaging Analysis}

Diffusion data were processed using the tools implemented in FSL (Version 5.0; www.fmrib.ox.ac.uk/fslwww.fmrib.ox.ac.uk/ fsl). Diffusion date were first corrected for eddy current distortions and motion artifacts, then averaged to improve signal-tonoise ratio (17) and subsequently skull stripped (using FSL's brain extraction tool). Non-diffusion-weighted images (B0) were also utilized for field map correction to reduce geometric distortions. Each participant's fractional anisotropy (FA) image was subsequently normalized into Montreal Neurological Institute (MNI) space via linear registration and Fourier interpolation through the FMRIB linear image registration tool. 


\section{Interhemispheric Callosal Tractography}

Probabilistic fiber tractography to assess quantity and quality of interhemispheric structural connectivity for the body of the corpus callosum was carried out (Figure 1A). Similar to previous work $(8,18)$, we utilized a multiple ROI approach to provide specific fiber tract identification of callosal fibers connecting the primary and secondary sensorimotor areas. First, the Human Motor Area Template, which identifies the primary and secondary sensorimotor regions of the cortices (19), was co-registered to each individual's MNI-normalized FA image and then used as a mask (20). The HMAT is an oft-used sensorimotor template that was identified through a meta-analysis examining functional MRI-defined cortical activity. Four sensorimotor regions were subsequently used to identify homologous, transcallosal fiber tracts connecting the SMAs and pre-SMAs, respectively, as well as the primary motor (M1) and the primary somatosensory (S1) cortices. In addition, for each interhemispheric sensorimotor fiber tract we utilized a "waypoint" ROI within the corresponding region of the body of the corpus callosum as identified by previous work (Figure 1B) (20).

For all interhemispheric sensorimotor tracts, probabilistic fiber tracking was initiated from every voxel within the binarized cortical seed HMAT ROI in each participant's native diffusion space, was required to pass through the corresponding callosal ROI waypoint, and terminated in the contralateral hemisphere's homologous regions ROI. We identified four distinct interhemispheric fiber tracts connecting the: (1) pre = SMA, (2) SMA, (3) M1, and (4) S1, respectively. Due to the difficulty in delineating differences between the interhemispheric connections between the ventral and dorsal premotor cortices (20), we choose to omit these ROIs from the current analysis.

As previously described (21), implicitly modeling noise in a probabilistic model allows for fiber tracking without externally added constraints such as FA threshold or fiber angle. As a result, fiber tracking in or near cortical areas, as in this study, becomes more sensitive. Utilizing a two-fiber model (22), as in this study, also improves the identification of crossing fibers.
For all tractography, a large number of samples $(25,000)$ were initiated from each voxel within the HMAT mask with the following parameters: step length of $0.5 \mathrm{~mm}$, curvature threshold of 0.2. Next, the probabilistic fibers were thresholded on individual maps to include voxels with a minimum of $50 \%$ of samples [i.e., selecting all connections where $>12,500$ of 25,000 samples passed; a very conservative level in comparison to previous work using a threshold of $5 \%(20,23)]$. Finally, the identified fiber tracts were binarized and affine-transferred into MNI space and summed across participants. Analysis of tract volume and FA was calculated for all tracts identified within the four interhemispheric pathways of interest, and analysis was subsequently restricted to each participant's 10 mid-sagittal slices ( \pm 5 slices from the mid-sagittal slice) to ensure that analysis was restricted to fiber tracts housed entirely within the corpus callosum.

\section{Statistical Analysis}

Diffusion derived metrics were compared $v i a$ a repeated measures analysis of variance (2 groups $\times 4$ tracts). Larger FA values are indicative of greater directional diffusivity, which is traditionally interpreted as better white matter microstructure, e.g., denser axonal packing and higher levels of myelination $(21,22)$. Cohen's $d$ effect sizes to demonstrate the strength of group differences were calculated for all primary gait and fiber tract outcomes. Finally, fiber tract integrity of the four transcallosal fiber tracts were correlated with both spatial and temporal gait asymmetry in people with PD and $\mathrm{HC}$, respectively. Correlations were Bonferroni-corrected for multiple comparisons and considered significant if $\alpha \leq 0.05 / 2$.

\section{RESULTS}

\section{Mobility Performance}

Many people with PD walked with greater temporal and spatial gait asymmetry than age-matched healthy controls (Figure 2).
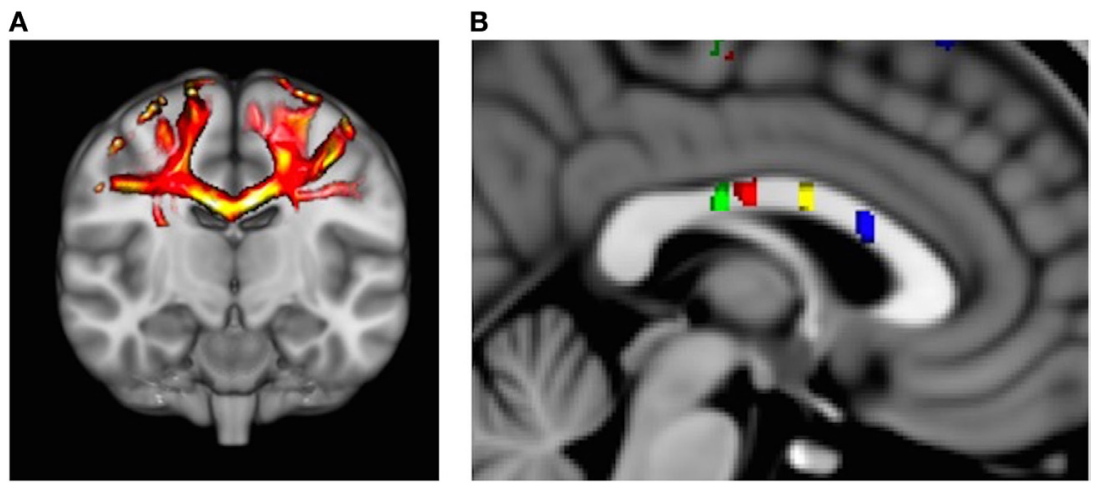

FIGURE 1 | (A) Representative example of identified transcallosal fiber tracts connecting the homologous right and left primary motor (M1) in a participant with Parkinson's disease. (B) Analysis of white matter tract microstructure was subsequently restricted to regions of interest identified on 10 mid-sagittal slices contained within the corpus callosum. Green = tracts connecting primary somatosensory; red = tracts connecting $\mathrm{M} 1$; yellow = tracts connecting supplementary motor area $(\mathrm{SMA})$; and blue = tracts connecting pre-SMA. 

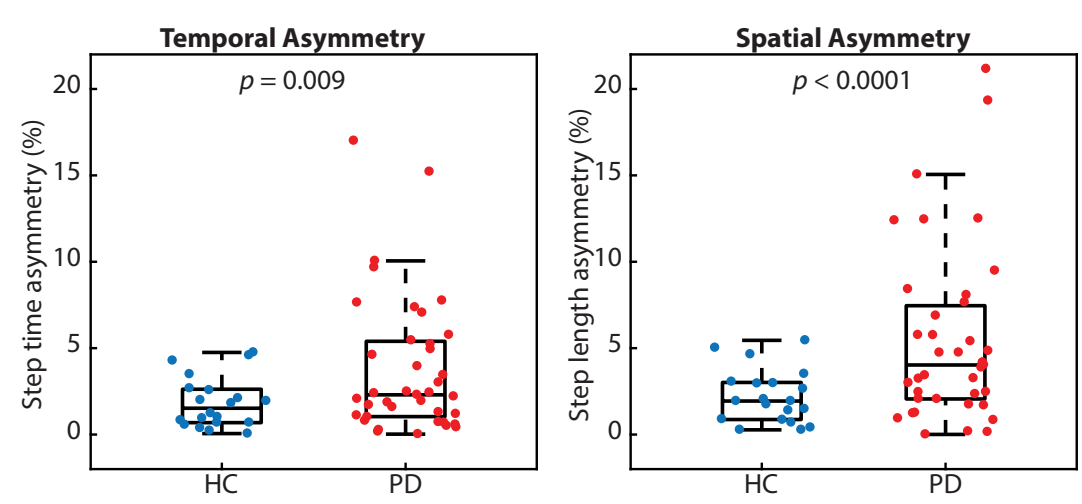

FIGURE 2 | People with Parkinson's disease (PD) exhibited significantly more temporal and spatial gait asymmetry than healthy controls $(p=0.009$ and $p<0.0001$ respectively).

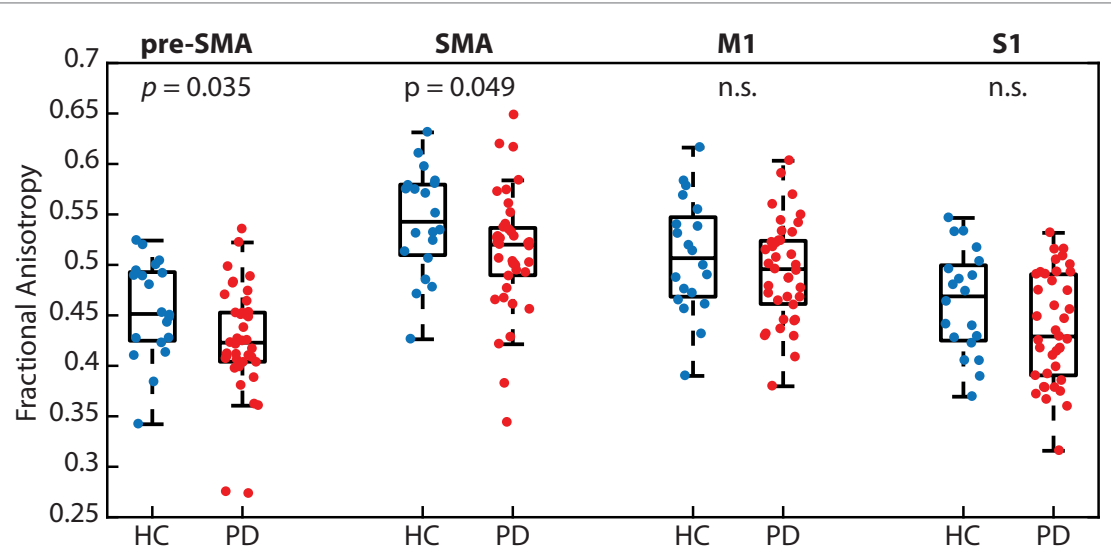

FIGURE 3 | People with Parkinson's disease (PD) had significantly reduced white matter microstructural integrity of the transcallosal fibers connecting homologous regions of the pre-supplementary motor area (SMA) and SMA, but not fibers connecting the primary motor (M1) and primary somatosensory (S1) cortices, when compared with age-matched control participants.

While both metrics of asymmetry were greater in people with $\mathrm{PD}$, we report a stronger effect size for spatial asymmetry $(d=0.78)$ as compared with temporal asymmetry $(d=0.59)$. People with PD also walked slower $(p<0.001$; PD $1.05 \pm 0.19 \mathrm{~m} / \mathrm{s}$ and HC $1.28 \pm 0.16 \mathrm{~m} / \mathrm{s})$ and with a shorter step length $(p<0.001 ; \mathrm{PD}$ $0.55 \pm 0.09 \mathrm{~m}$ and HC $0.66 \pm 0.06 \mathrm{~m}$ ), but similar step time $(p<0.48$; PD $0.53 \pm 0.05 \mathrm{~s}$ and HC $52 \pm 0.04 \mathrm{~s})$ compared with healthy control subjects.

\section{Transcallosal Fiber Tract Integrity}

People with PD had significantly reduced white matter microstructural integrity (i.e., FA) of transcallosal fibers connecting homologous regions of the pre-SMA $(p<0.05$; PD $0.42 \pm 0.05$ and HC $0.46 \pm 0.05)$ and SMA $(p<0.05$; PD $0.51 \pm 0.06$ and HC $0.54 \pm 0.05)$, but not M1 $(p=0.34)$ or S1 $(p=0.09$; Figure 3$)$. We refer the reader to Table 2 for effect sizes comparing the strength of group differences across the four fiber tracts, which ranged from small (0.28) to medium (0.59).
TABLE 2 | Cohen's $d$ effect sizes calculated for the primary gait and fiber tract sizes between groups.

\begin{tabular}{lc}
\hline & Cohen's $\mathbf{d}$ \\
\hline Temporal asymmetry & 0.59 \\
Spatial asymmetry & 0.78 \\
Pre-supplementary motor area (SMA) & 0.59 \\
SMA & 0.54 \\
Primary motor & 0.28 \\
Primary somatosensory & 0.48
\end{tabular}

A large effect $(\geq 0.75$ and $<1.10)$; medium effect $(\geq 0.40$ and $<0.75)$; and small effect $(\geq 0.15$ and $<0.40)$.

\section{Associations Between Gait Asymmetry and Transcallosal Fiber Tract Integrity}

Poorer transcallosal tract integrity of fibers connecting the preSMA $(r=-0.58 ; p<0.001)$ was associated with greater step length asymmetry in people with $\mathrm{PD}$, but not in healthy controls 

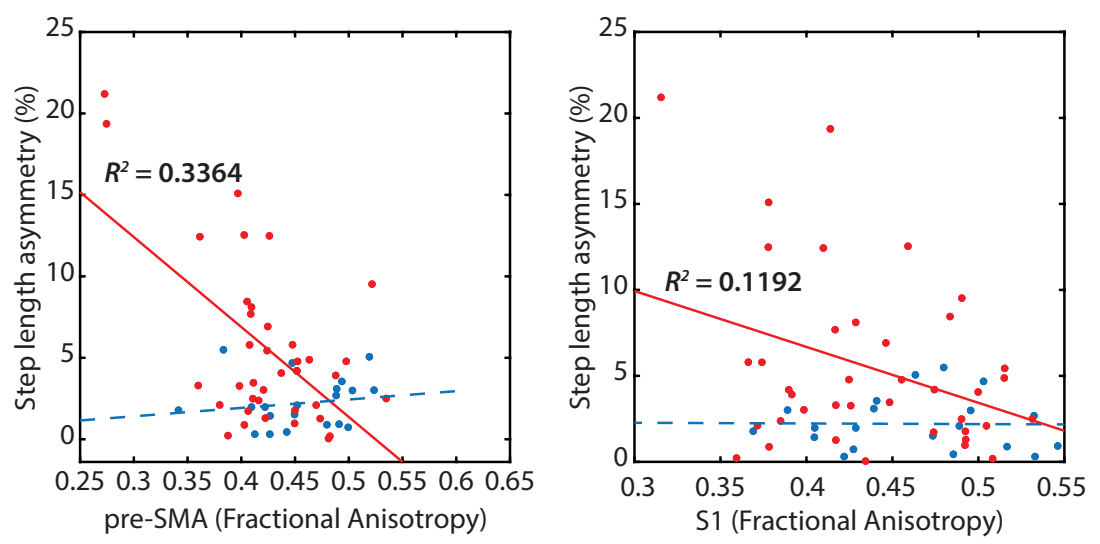

FIGURE 4 | Reduced transcallosal fiber tract integrity of the pre-supplementary motor area (SMA) and primary somatosensory (S1) were associated with greater step length asymmetry in people with Parkinson's disease, but not in healthy controls.

TABLE 3 | Correlation between the microstructural integrity of the callosal sensorimotor regions and spatial and temporal gait asymmetry (correlations with $p<0.05$ are highlighted in bold).

\begin{tabular}{|c|c|c|c|c|}
\hline & \multicolumn{2}{|c|}{ Spatial asymmetry } & \multicolumn{2}{|c|}{$\begin{array}{c}\text { Temporal } \\
\text { asymmetry }\end{array}$} \\
\hline & $\begin{array}{c}\text { Parkinson's disease } \\
\text { (PD) }\end{array}$ & $\mathrm{HC}$ & PD & $\mathrm{HC}$ \\
\hline $\begin{array}{l}\text { Pre-supplementary } \\
\text { motor area (SMA) }\end{array}$ & -0.5800 & 0.1581 & -0.0780 & 0.3425 \\
\hline SMA & -0.2699 & 0.2328 & -0.0480 & 0.3744 \\
\hline Primary motor & -0.1367 & -0.1162 & 0.1924 & 0.3813 \\
\hline Primary somatosensory & -0.3452 & -0.0123 & 0.1923 & 0.0758 \\
\hline
\end{tabular}

(Figure 4). In addition, FA of fiber tracts interhemispherically connecting the right and left S1 was strongly associated with spatial gait asymmetry in people with $\mathrm{PD}$, although not significant when corrected for multiple comparisons $(r=-0.34 ; p=0.03)$. No significant association was observed between spatial asymmetry and tract integrity of fibers connecting either the SMA or the M1 in people with $\mathrm{PD}$. In addition, no correlations were observed between temporal asymmetry and any of the four transcallosal fiber tracts $(r<0.19$ for all) in those with PD nor were any significant correlations observed between fiber tract integrity and gait asymmetry for healthy control subjects (Table 3 ).

\section{DISCUSSION}

Differences in gait asymmetry between those with PD and HC were observed both spatially and temporally. Individuals with PD also had significantly reduced microstructural integrity of white matter fibers connecting the right and left pre-SMA and right and left SMA, regions responsible for higher-order motor control. Conversely, no differences in interhemispheric fiber integrity were found for those tracts connecting the right and left primary motor or somatosensory cortices. Finally, impaired neuroanatomy connecting the right and left higher-order motor planning regions of the sensorimotor cortical hemispheres
(pre-SMA) and the S1 cortices resulted in a reduced capacity for spatially coordinating and controlling the legs during gait, specifically in people with $\mathrm{PD}$.

\section{Mobility Findings}

While gait is generally considered to be symmetric, subtle asymmetries do exist, even in HC (24). Findings on gait asymmetry in $\mathrm{PD}$ are inconsistent, while some studies have shown that gait asymmetry is increased in people with PD, especially in PD who experience freezing of gait $(25,26)$ others found no difference in spatial or temporal step asymmetry during over-ground walking (27). Notably, while step length asymmetry has previously been shown to be weakly correlated with disease severity (27), but not associated with asymmetry of clinical motor symptoms (25). Similar to spatial gait asymmetry (25), temporal gait asymmetry has not been found to be associated with laterality of motor symptom presentation of the disease $(25,28)$. Our current results demonstrate significantly increased spatial (e.g., step length) and temporal (e.g., step time) gait asymmetry in people with $\mathrm{PD}$, when measured in the OFF levodopa state, when gait is most affected. While previous work has offered descriptive metrics of gait asymmetry in people with $\mathrm{PD}$, the current work also provides evidence for potential neural bases underlying this altered gait pattern that appears to be independent of disease laterality as typically assessed by clinical motor assessments.

\section{Neuroimaging Findings}

A recent meta-analysis by Atkinson-Clement et al. (15) reports consistent and significant reductions in white matter macrostructural integrity of the corpus callosum, as assessed by FA, in people with PD compared with age-matched healthy control subjects (15). While the individual contributions of axonal density and myelination to FA are not fully understood, recent work indicates that axonal membranes likely play the primary role, whereas myelination can modulate the degree of anisotropy (29). As an example, anisotropy is reduced in demyelinating disease [multiple sclerosis (30)] and in conditions of premyelination [children (31)]. While several studies have focused on the 
anterior portions of the callosum (i.e., the genu) and its relation to cognitive decline in those with PD (32-34), there is a small, but growing body of literature indicating associations between callosal integrity and locomotor control in PD and the elderly $(8,35)$.

Interhemispheric communication via the corpus callosum is a well-known contributor to coordinated bimanual upper extremity control in healthy $(10,36,37)$ and neurodegenerative $(30,38)$ populations. Specifically, intact transcallosal structure has been shown to prevent interference between the two hands, particularly during bimanual out-of-phase actions (similar to gait) as compared with bimanual simultaneous movements (36, $39,40)$. Providing further confirmation for the specific relationship between transcallosal connectivity and asymmetric bilateral control, those patients who have received a callosotomy maintain the capacity to synchronously coordinate their two hands while performing discrete, simultaneous actions (41). This finding indicates that bilateral coordination remains possible, via pathways distinct from callosal communication. The current work extends this relationship between integrity of transcallosal sensorimotor fiber tracts to bilateral, out-of-phase control of the lower extremities in a large sample of people with PD. Specifically, these results point to transcallosal connections between the pre-SMA and the $\mathrm{S} 1$ cortices as important transcallosal fiber tracts associated with reduced symmetric control of gait in PD. Somatosensory cortices and medial motor areas like the pre-SMA have oft been implicated as serving prominent roles during complex bilateral movements.

Gerloff and Andres (42) have previously identified a complex cortical network underlying bimanual coordination, and they highlight the importance of the bilateral primary sensorimotor cortices, along with medial motor wall areas including the cingulate motor area and the pre-SMA. There are particularly dense homotopic transcallosal connections within the pre-SMA and SMAs (43), and these higher-order motor regions substantially influence M1 activity in both hemispheres during the execution of visually paced movements (44). For example, the pre-SMA significantly inhibits the opposite hemisphere's M1, thereby suppressing its activity. A growing body of literature investigating the effects of non-invasive brain stimulation (e.g., repetitive TMS) to reduce activity of the pre-SMA and SMA has shown that temporal pacing while bimanually tapping the fingers in an anti-phase pattern is selectively degraded, as compared with synchronously tapping (45). The authors suggest that deterioration of interhemispheric coupling due to stimulation likely reduces interhemispheric inhibition resulting in poorer motor performance on tasks requiring a higher level of interhemispheric inhibition. Similarly, our current results demonstrate that impaired neuroanatomy connecting the bilateral pre-SMAs results in a decreased ability to produce consistent steps with regards to the spatial domain in individuals with PD.

While the current literature is quite limited with regards to interhemispheric transfer of information between the primary somatosensory cortices, Geffen and colleagues (46) report that afferent feedback carries significant information regarding temporal control of movement and that it is a reduction in this sensory feedback that primarily impairs out-of-phase bilateral movement such as gait. Furthermore, recent work from Jung et al. (47) demonstrates that interhemispheric inhibition transmitted transcallosally between the bilateral somatosensory cortices is directly correlated with bimanual tactile performance, indicating that these interhemispheric sensory fiber tracts have behavioral importance for bimanual object manipulation and exploration. Structural findings within the brains of pianist's, a group that demands exquisite bilateral control and coordination, also demonstrates structural adaptations/enlargements of the SI hand representation (48). The current results are the first we are aware of that demonstrate the importance of transcallosal somatosensory fiber tracts and the ability to output consistent spatial bilateral gait patterns (i.e., steps). Collectively, the pre-SMA and S1 both exert an inhibitory influence on the contralateral M1, particularly for tasks where each limb undergoes independent spatiotemporal profiles that must be coordinated together, like gait in this study.

A limitation of the current manuscript is the amount of gait captured, comprising only three trials over an 8-m walkway per participant. While the use of an instrumented walkway is required to accurately assess spatial asymmetry during typical gait, this approach has been shown to be reflective of multiple gait characteristics including gait speed, cadence, and step length and time variables, as used in this study (49).

\section{CONCLUSION}

People with PD showed greater temporal and spatial gait asymmetry between the two legs along with decreased microstructural integrity of callosal white matter tracts connecting the pre-SMA and SMAs. Furthermore, strong associations were observed between callosal integrity of fiber tracts connecting pre-SMA and S1 cortical regions and step length asymmetries, solely in people with PD. These results indicate that reduced transcallosal sensorimotor structural connectivity may be a significant mechanism underlying bilateral gait asymmetries in those with PD.

\section{ETHICS STATEMENT}

All patients or their next of kin gave informed, written consent to a protocol approved by the Institutional Review Board of Oregon Health and Science University.

\section{AUTHOR CONTRIBUTIONS}

$\mathrm{BF}, \mathrm{FH}$, and $\mathrm{CC}$ all contributed to the conceptual design and interpretation of this study. BF and CC contributed to data collection and analysis.

\section{FUNDING}

This work was supported by the National Institutes of Health (2R01AG006457; KL2TR000152), the Department of Veterans Affairs grant \# I01RX001075, the Collins Medical Trust, and the Medical Research Foundation of Oregon. 


\section{REFERENCES}

1. Jankovic J. Parkinson's disease: clinical features and diagnosis. JNeurol Neurosurg Psychiatry (2008) 79(4):368-76. doi:10.1136/jnnp.2007.131045

2. Morris ME, Matyas TA, Iansek R, Summers JJ. Temporal stability of gait in Parkinson's disease. Phys Ther (1996) 76(7):763-77; discussion 778-80. doi:10.1093/ptj/76.7.763

3. Almeida QJ, Frank JS, Roy EA, Patla AE, Jog MS. Dopaminergic modulation of timing control and variability in the gait of Parkinson's disease. Mov Disord (2007) 22(12):1735-42. doi:10.1002/mds.21603

4. Platts MM, Rafferty D, Paul L. Metabolic cost of over ground gait in younger stroke patients and healthy controls. Med Sci Sports Exerc (2006) 38(6):1041-6. doi:10.1249/01.mss.0000222829.34111.9c

5. Stoquart G, Detrembleur C, Lejeune TM. The reasons why stroke patients expend so much energy to walk slowly. Gait Posture (2012) 36(3):409-13. doi:10.1016/j.gaitpost.2012.03.019

6. Finley JM, Bastian AJ. Associations between foot placement asymmetries and metabolic cost of transport in hemiparetic gait. Neurorehabil Neural Repair (2017) 31(2):168-77. doi:10.1177/1545968316675428

7. Wang HC, Hsu JL, Leemans A. Diffusion tensor imaging of vascular parkinsonism: structural changes in cerebral white matter and the association with clinical severity. Arch Neurol (2012) 69(10):1340-8. doi:10.1001/ archneurol.2012.633

8. Fling BW, Dale ML, Curtze C, Smulders K, Nutt JG, Horak FB. Associations between mobility, cognition and callosal integrity in people with parkinsonism. Neuroimage Clin (2016) 11:415-22. doi:10.1016/j.nicl.2016. 03.006

9. Dale ML, Mancini M, Curtze C, Horak FB, Fling BW. Freezing of gait associated with a corpus callosum lesion. J Clin Mov Disord (2016) 3:2. doi:10.1186/ s40734-016-0030-2

10. Fling BW, Seidler RD. Fundamental differences in callosal structure, neurophysiologic function, and bimanual control in young and older adults. Cereb Cortex (2012) 22(11):2643-52. doi:10.1093/cercor/bhr349

11. Gooijers J, Swinnen SP. Interactions between brain structure and behavior: the corpus callosum and bimanual coordination. Neurosci Biobehav Rev (2014) 43:1-19. doi:10.1016/j.neubiorev.2014.03.008

12. Sohn YH, Jung HY, Kaelin-Lang A, Hallett M. Excitability of the ipsilateral motor cortex during phasic voluntary hand movement. Exp Brain Res (2003) 148(2):176-85. doi:10.1007/s00221-002-1292-5

13. Stinear CM, Byblow WD. Role of intracortical inhibition in selective hand muscle activation. J Neurophysiol (2003) 89(4):2014-20. doi:10.1152/ jn.00925.2002

14. Hall JM, Ehgoetz Martens KA, Walton CC, O'Callaghan C, Keller PE, Lewis SJ, et al. Diffusion alterations associated with Parkinson's disease symptomatology: a review of the literature. Parkinsonism Relat Disord (2016) 33:12-26. doi:10.1016/j.parkreldis.2016.09.026

15. Atkinson-Clement C, Pinto S, Eusebio A, Coulon O. Diffusion tensor imaging in Parkinson's disease: review and meta-analysis. Neuroimage Clin (2017) 16:98-110. doi:10.1016/j.nicl.2017.07.011

16. Fling BW, Cohen RG, Mancini M, Carpenter SD, Fair DA, Nutt JG, et al. Functional reorganization of the locomotor network in Parkinson patients with freezing of gait. PLoS One (2014) 9(6):e100291. doi:10.1371/journal. pone. 0100291

17. Eickhoff SB, Jbabdi S, Caspers S, Laird AR, Fox PT, Zilles K, et al. Anatomical and functional connectivity of cytoarchitectonic areas within the human parietal operculum. J Neurosci (2010) 30(18):6409-21. doi:10.1523/ JNEUROSCI.5664-09.2010

18. Fling BW, Benson BL, Seidler RD. Transcallosal sensorimotor fiber tract structure-function relationships. Hum Brain Mapp (2013) 34(2):384-95. doi: $10.1002 / \mathrm{hbm} .21437$

19. Mayka MA, Corcos DM, Leurgans SE, Vaillancourt DE. Three-dimensional locations and boundaries of motor and premotor cortices as defined by functional brain imaging: a meta-analysis. Neuroimage (2006) 31(4):1453-74. doi:10.1016/j.neuroimage.2006.02.004

20. Fling BW, Cohen RG, Mancini M, Nutt JG, Fair DA, Horak FB. Asymmetric pedunculopontine network connectivity in parkinsonian patients with freezing of gait. Brain (2013) 136(Pt 8):2405-18. doi:10.1093/brain/ awt172
21. Behrens TE, Woolrich MW, Jenkinson M, Johansen-Berg H, Nunes RG, Clare $\mathrm{S}$, et al. Characterization and propagation of uncertainty in diffusion-weighted MR imaging. Magn Reson Med (2003) 50(5):1077-88. doi:10.1002/mrm. 10609

22. Behrens TE, Berg HJ, Jbabdi S, Rushworth MF, Woolrich MW. Probabilistic diffusion tractography with multiple fibre orientations: what can we gain? Neuroimage (2007) 34(1):144-55. doi:10.1016/j.neuroimage.2006.09.018

23. Gschwind M, Pourtois G, Schwartz S, Van De Ville D, Vuilleumier P. Whitematter connectivity between face-responsive regions in the human brain. Cereb Cortex (2012) 22(7):1564-76. doi:10.1093/cercor/bhr226

24. Sadeghi H, Allard P, Prince F, Labelle H. Symmetry and limb dominance in able-bodied gait: a review. Gait Posture (2000) 12(1):34-45. doi:10.1016/ S0966-6362(00)00070-9

25. Plotnik M, Giladi N, Balash Y, Peretz C, Hausdorff JM. Is freezing of gait in Parkinson's disease related to asymmetric motor function? Ann Neurol (2005) 57(5):656-63. doi:10.1002/ana.20452

26. Plotnik M, Giladi N, Hausdorff JM. A new measure for quantifying the bilateral coordination of human gait: effects of aging and Parkinson's disease. Exp Brain Res (2007) 181(4):561-70. doi:10.1007/s00221-007-0955-7

27. Nanhoe-Mahabier W, Snijders AH, Delval A, Weerdesteyn V, Duysens $\mathrm{J}$, Overeem S, et al. Walking patterns in Parkinson's disease with and without freezing of gait. Neuroscience (2011) 182:217-24. doi:10.1016/j. neuroscience.2011.02.061

28. Yogev G, Plotnik M, Peretz C, Giladi N, Hausdorff JM. Gait asymmetry in patients with Parkinson's disease and elderly fallers: when does the bilateral coordination of gait require attention? Exp Brain Res (2007) 177(3):336-46. doi:10.1007/s00221-006-0676-3

29. Gulani V, Webb AG, Duncan ID, Lauterbur PC. Apparent diffusion tensor measurementsinmyelin-deficientratspinalcords.MagnResonMed(2001)45(2):1915. doi:10.1002/1522-2594(200102)45:2<191::AID-MRM1025>3.0.CO;2-9

30. Bonzano L, Tacchino A, Roccatagliata L, Abbruzzese G, Mancardi GL, Bove M. Callosal contributions to simultaneous bimanual finger movements. J Neurosci (2008) 28(12):3227-33. doi:10.1523/JNEUROSCI.4076-07.2008

31. Barnea-Goraly N, Menon V, Eckert M, Tamm L, Bammer R, Karchemskiy A, et al. White matter development during childhood and adolescence: a cross-sectional diffusion tensor imaging study. Cereb Cortex (2005) 15(12):1848-54. doi:10.1093/cercor/bhi062

32. Kamagata K, Motoi Y, Tomiyama H, Abe O, Ito K, Shimoji K, et al. Relationship between cognitive impairment and white-matter alteration in Parkinson's disease with dementia: tract-based spatial statistics and tract-specific analysis. Eur Radiol (2013) 23(7):1946-55. doi:10.1007/s00330-013-2775-4

33. Zheng Z, Shemmassian S, Wijekoon C, Kim W, Bookheimer SY, Pouratian N. DTI correlates of distinct cognitive impairments in Parkinson's disease. Hum Brain Mapp (2014) 35(4):1325-33. doi:10.1002/hbm.22256

34. Deng B, Zhang Y, Wang L, Peng K, Han L, Nie K, et al. Diffusion tensor imaging reveals white matter changes associated with cognitive status in patients with Parkinson's disease. Am J Alzheimers Dis Other Demen (2013) 28(2):154-64. doi:10.1177/1533317512470207

35. Chan LL, Ng KM, Rumpel H, Fook-Chong S, Li HH, Tan EK. Transcallosal diffusion tensor abnormalities in predominant gait disorder parkinsonism. Parkinsonism Relat Disord (2014) 20(1):53-9. doi:10.1016/j. parkreldis.2013.09.017

36. Fling BW, Seidler RD. Task-dependent effects of interhemispheric inhibition on motor control. Behav Brain Res (2012) 226(1):211-7. doi:10.1016/j. bbr.2011.09.018

37. Fling BW, Walsh CM, Bangert AS, Reuter-Lorenz PA, Welsh RC, Seidler RD. Differential callosal contributions to bimanual control in young and older adults. J Cogn Neurosci (2011) 23(9):2171-85. doi:10.1162/jocn.2010.21600

38. Bonzano L, Tacchino A, Roccatagliata L, Mancardi GL, Abbruzzese G, Bove M. Structural integrity of callosal midbody influences intermanual transfer in a motor reaction-time task. Hum Brain Mapp (2011) 32(2):218-28. doi:10.1002/hbm. 21011

39. Stinear JW, Byblow WD. Disinhibition in the human motor cortex is enhanced by synchronous upper limb movements. J Physiol (2002) 543(Pt 1):307-16. doi:10.1113/jphysiol.2002.023986

40. Swinnen SP. Intermanual coordination: from behavioural principles to neural-network interactions. Nat Rev Neurosci (2002) 3(5):348-59. doi:10.1038/ nrn807 
41. Kennerley SW, Diedrichsen J, Hazeltine E, Semjen A, Ivry RB. Callosotomy patients exhibit temporal uncoupling during continuous bimanual movements. Nat Neurosci (2002) 5(4):376-81. doi:10.1038/nn822

42. Gerloff C, Andres FG. Bimanual coordination and interhemispheric interaction. Acta Psychol (Amst) (2002) 110(2-3):161-86. doi:10.1016/ S0001-6918(02)00032-X

43. Rouiller EM, Babalian A, Kazennikov O, Moret V, Yu XH, Wiesendanger M. Transcallosal connections of the distal forelimb representations of the primary and supplementary motor cortical areas in macaque monkeys. Exp Brain Res (1994) 102(2):227-43. doi:10.1007/BF00227511

44. Grefkes C, Eickhoff SB, Nowak DA, Dafotakis M, Fink GR. Dynamic intra- and interhemispheric interactions during unilateral and bilateral hand movements assessed with fMRI and DCM. Neuroimage (2008) 41(4):1382-94. doi:10.1016/j.neuroimage.2008.03.048

45. Serrien DJ, Strens LH, Oliviero A, Brown P. Repetitive transcranial magnetic stimulation of the supplementary motor area (SMA) degrades bimanual movement control in humans. Neurosci Lett (2002) 328(2):89-92. doi:10.1016/ S0304-3940(02)00499-8

46. Geffen GM, Jones DL, Geffen LB. Interhemispheric control of manual motor activity. Behav Brain Res (1994) 64(1-2):131-40. doi:10.1016/0166-4328(94)90125-2
47. Jung P, Klein JC, Wibral M, Hoechstetter K, Bliem B, Lu MK, et al. Spatiotemporal dynamics of bimanual integration in human somatosensory cortex and their relevance to bimanual object manipulation. J Neurosci (2012) 32(16):5667-77. doi:10.1523/JNEUROSCI.5957-11.2012

48. Gaser C, Schlaug G. Brain structures differ between musicians and non-musicians. J Neurosci (2003) 23(27):9240-5.

49. Webster KE, Wittwer JE, Feller JA. Validity of the GAITRite walkway system for the measurement of averaged and individual step parameters of gait. Gait Posture (2005) 22(4):317-21. doi:10.1016/j.gaitpost.2004.10.005

Conflict of Interest Statement: The authors declare that the research was conducted in the absence of any commercial or financial relationships that could be construed as a potential conflict of interest.

Copyright ( $(2) 18$ Fling, Curtze and Horak. This is an open-access article distributed under the terms of the Creative Commons Attribution License (CC BY). The use, distribution or reproduction in other forums is permitted, provided the original author(s) and the copyright owner are credited and that the original publication in this journal is cited, in accordance with accepted academic practice. No use, distribution or reproduction is permitted which does not comply with these terms. 